\title{
Geoneutrinos in Borexino
}

\author{
Marco G. Giammarchi and Lino Miramonti ${ }^{1}$ \\ Dipartimento di Fisica dell'Università di Milano and Infn. \\ Via Celoria 16, 20133 Milano, Italy
}

\begin{abstract}
This paper describes the Borexino detector and the high-radiopurity studies and tests that are integral part of the Borexino technology and development. The application of Borexino to the detection and studies of geoneutrinos is discussed.
\end{abstract}

\section{The Gran Sasso National Laboratory}

The Gran Sasso National Laboratory (Laboratori Nazionali del Gran Sasso - LNGS), home of the Borexino experiment, is the world's largest underground laboratory. It is located in the center of Italy in the highway tunnel between Teramo and L'Aquila under the "Monte Aquila" (Gran Sasso mountain). The laboratory is financed and operated by the Italian National Institute for Nuclear Physics (Infn). Its total underground volume is about $180,000 \mathrm{~m}^{3}$ with an area greater than $13500 \mathrm{~m}^{2}$. It is composed of three main experimental halls $(20 \mathrm{~m}$ high, $18 \mathrm{~m}$ wide and 100 $\mathrm{m}$ long). The overburden rock is on the average about $1,400 \mathrm{~m}$, equivalent to 3,700 meters of water. The muon flux is reduced by about 6 orders of magnitude to a value of approximately 1.1 muons per square meter per hour, whereas the neutron flux is of the order of $3 \times 10^{-6}$ neutrons per square centimeter per second with energies greater than $2.5 \mathrm{MeV}$.

The rock of the Gran Sasso mountain has a density of $2.71 \pm 0.05 \mathrm{~g} \cdot \mathrm{cm}^{-3}$, and consists mainly of $\mathrm{CaCO}_{3}$ and $\mathrm{MgCO}_{3}$ 1]. The primordial radionuclide content of the rock of Hall $\mathrm{C}$ is $0.66 \pm 0.14 \mathrm{ppm}$ for ${ }^{238} \mathrm{U}, 0.066 \pm 0.025 \mathrm{ppm}$ for the ${ }^{232} \mathrm{Th}$ and $160 \mathrm{ppm}$ for $\mathrm{K}$ [2. The radioactive content of the concrete employed as experimental hall liner is $1.05 \pm 0.12 \mathrm{ppm}$ for ${ }^{238} \mathrm{U}$ and $0.656 \pm 0.028 \mathrm{ppm}$ for the ${ }^{232} \mathrm{Th}[3]$.

The LNGS hosts about 15 experiments of astroparticle physiscs such as neutrino research, double beta decay physics, dark matter studies and nuclear astrophysics. Interdisciplinary studies (biology, geology) are also conducted in the LNGS underground location.

The Borexino detector is located in one of the big underground experimental halls, hall C.

\section{The Borexino detector}

Borexino is a real time experiment whose main goal is to study the low energy (sub-MeV) solar neutrinos, and in particular the $862 \mathrm{keV}{ }^{7} \mathrm{Be}$ solar neutrino line, through the neutrino-electron elastic scattering reaction. The maximum energy of the recoiling electron is $664 \mathrm{keV}$ and the experimental design threshold is set at $250 \mathrm{keV}$ [4].

1 talk given by M.G. Giammarchi 
Borexino is an unsegmented scintillation detector featuring 300 tonnes of well shielded liquid ultra-pure scintillator viewed by 2200 photomultipliers (PMT). The detector core is a transparent spherical vessel (Nylon Sphere, $100 \mu \mathrm{m}$ thick), $8.5 \mathrm{~m}$ of diameter, filled with 300 tonnes of liquid scintillator and surrounded by 1,000 tonnes of high-purity buffer liquid. The scintillator mixture is PC (Pseudocumene) and PPO (1.5 g/l) as a fluor, while the buffer liquid will be PC alone (with the addition of DMP as light quencher). The photomultipliers are supported by a Stainless Steel Sphere, which also separates the inner part of the detector from the external shielding, provided by 2400 tonnes of pure water (water buffer), see figure ??.

An additional containment vessel (Nylon film Radon barrier) is interposed between the Nylon Sphere and the photomultipliers, with the goal of reducing Radon diffusion towards the internal part of the detector.

The outer water shield is instrumented with 200 outward-pointing PMT's serving as a veto for penetrating muons, the only significant remaining cosmic ray background at the Gran Sasso depth.

The innermost 2200 photomultipliers are divided into a set of 1800 PMT's equipped with light cones (so that they see light only from the Nylon Sphere region) and a set of 400 PMT's without light cones, sensitive to light originated in the whole Stainless Steel Sphere volume. This design greatly increases the capability of the system to identify muons crossing the PC buffer (and not the scintillator).

The Borexino design is based on the concept of a graded shield of progressively lower intrinsic radioactivity as one approaches the sensitive volume of the detector; this culminates in the use of 200 tonnes of the low background scintillator to shield the 100 tonnes innermost Fiducial Volume. In these conditions, the ultimate background will be dominated by the intrinsic contamination of the scintillator, while all backgrounds from the construction materials and external shieldings will be negligible.

Borexino also features several external plants and purification systems conceived to purify the experimental fluids (water, nitrogen and scintillator) used by the experiment.

The main problem of a real time experiment with such a low energy threshold is the natural radioactivity which is present in any environment and in any material. For these reasons an intense R\&D program has been carried out in the last ten years to develop methods for selecting low radioactivity materials and/or purify them. An effort in this field has to be complemented by a comparably thorough research concerning detection and measurement of very low radioactivity levels. In this context four purification methods have been developed: distillation, water extraction, stripping with ultrapure $\mathrm{N}_{2}$, solid gel column ( $\mathrm{Si}$ gel, Al gel) adsorption.

Significative results have been achieved by the Collaboration as for example: $10^{-16}-10^{-17}$ (g of contaminants/g of material) for ${ }^{232} \mathrm{Th}$ and ${ }^{238} \mathrm{U}$ family and a few $\mu \mathrm{Bq}$ of $\mathrm{Rn}-222$ in gases and liquids. In addition the organic solvent selected by the collaboration showed a ${ }^{14} \mathrm{C}$ concentration clearly below $10^{-17}$ in its ratio to ${ }^{12} \mathrm{C}$; this impurity is particularly important because it cannot be removed by chemical purification processes.

For the measurements of these ultralow radioactivity levels, dedicated methods were developed. In addition to small-scale techniques (Ge underground detectors in Rn-free environments, Inductively Coupled Plasma Mass Spectometer, high sensitivity Neutron Activation, Atomic Absorption Spectroscopy etc... [5]) a prototype of the Borexino detector, the Counting Test Facility (CTF), has been constructed on purpose and operated in the Hall $\mathrm{C}$ of LNGS.

The radiopurities and sensitivities reached are summarized below and correspond to the lowest radioactivity levels obtained by the Borexino Collaboration, in preparation of the experiment:

- Bulk material radiopurities of $10^{-10} \mathrm{~g} / \mathrm{g}$ for ${ }^{238} \mathrm{U}$ and ${ }^{232} \mathrm{Th}, \sim 10^{-5}$ for ${ }^{n a t} \mathrm{~K}$, few tenths of $\mathrm{mBq} / \mathrm{kg}$ for ${ }^{60} \mathrm{Co}$, have been measured with Ge detectors in construction materials such as 
stainless steel, photomultipliers, metal and plastic gaskets, products for PMT sealing, etc...

- Radon emanations of $10 \mu \mathrm{Bq} / \mathrm{m}^{2}$ from plastic materials, $0.1 \mathrm{mBq} / \mathrm{m}^{3}$ for $\mathrm{Rn}-222$ and 1 $\mathrm{mBq} / \mathrm{m}^{3}$ for Ra-226 in water, below $1 \mathrm{mBq} / \mathrm{m}^{3}$ for the $\mathrm{N}_{2}$ used for scintillator stripping.

- Radiopurity levels of a few times $10^{-15} \mathrm{~g} / \mathrm{g}{ }^{238} \mathrm{U},{ }^{232} \mathrm{Th}$ and ${ }^{40} \mathrm{~K}$ have been reached with ICMPS in measuring the Borexino and CTF shielding water.

- Sensitivities of few ppt for ${ }^{238} \mathrm{U}$ and ${ }^{232} \mathrm{Th}$ concentrations have been obtained in the Nylon Sphere material measurements.

- The radiopurity of the scintillator itself was measured to be at the level of few $10^{-16} \mathrm{~g} / \mathrm{g}$ for ${ }^{238} \mathrm{U},{ }^{232} \mathrm{Th}$ and $\sim 10^{-18}$ for ${ }^{14} \mathrm{C} /{ }^{12} \mathrm{C}$ in the Counting Test Facility.

- Bulk radiopurity levels of $10^{-13}-10^{-14} \mathrm{~g} / \mathrm{g}$ for $\mathrm{Au}$, Ba, Ce, Co, Cr, Cs, Ga, Hg, In, Mo, $\mathrm{Rb}$; less than few $10^{-15} \mathrm{~g} / \mathrm{g}$ for $\mathrm{Cd}, \mathrm{Sb}, \mathrm{Ta}, \mathrm{W} ; 0^{-16}-10^{-17} \mathrm{~g} / \mathrm{g}$ for La, Lu, Re, Sc, Th; less than $1 \times 10^{-17} \mathrm{~g} / \mathrm{g}$ for $\mathrm{U}$, have been reached by means of Neutron Activation followed by $\beta-\gamma$ delayed coincidence analysis applied to the scintillator.

- $\mathrm{Kr}$ and Ar contamination in nitrogen at $0.005 \mathrm{ppm}$ (for Ar) and $0.06 \mathrm{ppt}$ (for $\mathrm{Kr}$ ) were obtained and measured with noble gas mass spectrometry.

These results represent a milestone in the development of the Borexino detector and technique. Several of these concepts were incorporated in the construction of the high purity systems for the treatment of the most critical liquid, the scintillator of the experiment.

\section{The Counting Test Facility}

The CTF description and its performance have been published elsewhere 6, 7, 8]. In this section we simply review the main features of this detector.

The CTF consists of an external cylindrical water tank $(\oslash 11 \times 10 \mathrm{~m} ; \simeq 1,000 \mathrm{t}$ of water $)$ serving as passive shielding for $4.8 \mathrm{~m}^{3}$ of liquid scintillator contained in an inner spherical vessel (Inner Vessel) of $2.1 \mathrm{~m}$ in diameter and observed by 100 PMT's. An additional nylon barrier against Radon convection and a muon veto system were installed in 1999. Figure ?? shows a picture of the CTF detector.

The radio-purity level of the water is $\simeq 10^{-14} \mathrm{~g} / \mathrm{g}(\mathrm{U}, \mathrm{Th}), \simeq 10^{-10} \mathrm{~g} / \mathrm{g}\left({ }^{\text {nat }} \mathrm{K}\right)$ and $<5$ $\mu \mathrm{Bq} / l$ for ${ }^{222} \mathrm{Rn}$ [6, 8, 9].

The organic liquid scintillator has the same composition as in Borexino. The yield of emitted photons is $\simeq 10^{4}$ per $\mathrm{MeV}$ of energy deposited and the fluorescence peak emission is located at $365 \mathrm{~nm}$. The principal scintillator decay time is $\simeq 3.5 \mathrm{~ns}$ in a small volume, while for large volume (because of absorbtion and re-emission) this value is $4.5-5.0 \mathrm{~ns}$. The attenuation length is larger than $5 \mathrm{~m}$ above $380 \mathrm{~nm}$ [10].

The purification of the scintillator is performed by recirculation from the Inner Vessel through a Radon stripping tower, a water extraction unit, a Si-Gel column extraction unit, and a vacuum distillation unit. The ${ }^{232} \mathrm{Th}$ and ${ }^{238} \mathrm{U}$ contaminations in the CTF liquid scintillator were found to be less than $(2-5) \cdot 10^{-16} \mathrm{~g} / \mathrm{g}$.

The Inner Vessel for the liquid scintillator containment is made of nylon with a thickness of $500 \mu \mathrm{m}$, with excellent optical clarity at $350-500 \mathrm{~nm}$. The collection of scintillation light is ensured by 100 PMT's mounted to a $7 \mathrm{~m}$ diameter support structure inside the CTF tank.

The photomultiplier tubes are 8 inches (Thorn EMI 9351, the same as for Borexino) made of low radioactivity Schott 8246 glass and characterized by high quantum efficiency $(26 \%$ at 420 $\mathrm{nm})$, limited transit time spread ( $\sigma=1 \mathrm{~ns})$, good pulse height resolution for single photoelectron pulses $($ Peak/Valley $=2.5)$, low dark noise rate $(0.5 \mathrm{kHz})$, low after pulse probability $(2.5 \%)$, and a gain of $10^{7}$. 
The PMT's are equipped with light concentrators $57 \mathrm{~cm}$ long and with $50 \mathrm{~cm}$ diameter aperture. The PMT system provides an overall $20 \%$ optical coverage for events taking place inside the Inner Vessel. The number of photoelectrons per $\mathrm{MeV}$ measured experimentally is $(300 \pm 30) / \mathrm{MeV}$ on average.

The total background rate in the $250-800 \mathrm{keV}$ energy range is about 0.3 counts $/ \mathrm{yr} \cdot \mathrm{keV} \cdot \mathrm{kg}$ and appears to be dominated by external background from Radon in the shielding water $(\approx 30$ $\mathrm{mBq} / \mathrm{m}^{3}$ in the region surrounding the Inner Vessel). The internal background was measured to be less than 0.01 counts $/ \mathrm{yr} \cdot \mathrm{keV} \cdot \mathrm{kg}$.

\section{The Counting Test Facility related publications}

Data collected with the Counting Test Facility have contributed significantly to the best limits on quantities such as neutrino magnetic moment, electron lifetime, nucleon decays in invisible channels, violation of the Pauli exclusion principle, production of heavy-neutrinos in the sun.

Concerning the study of the stability of the electron, the CTF data have been analyzed to search for the $256 \mathrm{keV}$ line of the gamma emitted in the decay channel $e \rightarrow \gamma \nu$. Since we have found no signal, we established a limit on the electron lifetime of $\tau \geq 4 \cdot 10^{26}$ (90\% C.L.); this is still the best world limit for the electron decay in this channel 11.

CTF data analysis has allowed the study of the neutrino magnetic moment, obtaining the limit of $\mu_{\nu} \leq 0.5 \cdot 10^{-10} \mu_{B}$, still a very competitive result [12].

We have also investigated the possibility of heavy neutrinos $\left(M \geq m_{e}\right)$ emitted in the ${ }^{8} B$ reaction in the sun. Heavy neutrinos would decay to light neutrinos via the reaction $\nu_{H} \rightarrow \nu_{L}+e^{+}+e^{-}$. The analysis of the $\mathrm{CTF}$ energy spectrum has allowed to significantly enlarge the excluded region of the parameter space with respect to previous experiments [13.

The stability of nucleons bounded in nuclei has been studied in the Counting Test Facility searching for decays of single nucleon or pair of nucleons into invisible channels. The limits are comparable to or improve the previously set world limits [14]. Furthermore a search was made for non-Paulian transitions of nucleons from nuclear $1 P$ shell to a filled $1 S_{1 / 2}$ shell obtaining the best limit on the Pauli exclusion principle [15].

Other studies have concerned the search for anti-neutrinos coming from the sun [16] and the cosmogenic ${ }^{11} \mathrm{C}$ underground production [17.

\section{Geoneutrinos detection}

One of the possible application of a high mass well shielded scintillator detector such as Borexino is the search for geoneutrinos, a new and very interesting subject which we will discuss in the remaining of this paper.

The conceptual foundations of Earth science rest on a variety of observables as well as interior

characteristics. One of the most important interior parameter is the internally produced heat which is currently measured to be in the $\sim 60 \mathrm{~mW} / \mathrm{m}^{2}$ range (or $30 \mathrm{TW}$ when integrated on the planet surface).

Part of this energy flow is due to the presence of radioactive elements in the Earth interior, mainly naturally occurring Uranium and Thorium chain elements and potassium. Models of the Earth disperse about $50 \%$ of the total U,Th in the crust while leaving the remaining half to the mantle. Roughly speaking, the $35 \mathrm{~km}$ thick continental crust contains a few ppm of U,Th while the much thinner $(\sim 6 \mathrm{~km})$ oceanic crust has a typical concentration of $\sim 0.1 \mathrm{ppm}$.

Our goal is to measure this radiogenic heat by detecting neutrinos emitted during the decays of the radioactive chains. For a given structure of naturally occurring radioactive families, a measurement of antineutrino flux can be related to the U,Th family content of the Earth. 
In the case of the ${ }^{238} \mathrm{U}$ family (the ${ }^{235} \mathrm{U}$ leftover can be neglected) one can globally represent the full decay chain as:

$$
{ }^{238} \mathrm{U} \rightarrow{ }^{206} \mathrm{~Pb}+8 \alpha+6 e^{-}+6 \bar{\nu}_{e}+51.7 \mathrm{MeV}
$$

and therefore the detected number of antineutrinos is related to the number of times this reaction has taken place.

The ${ }^{232}$ Th family presents a similar case:

$$
{ }^{232} \mathrm{Th} \rightarrow{ }^{208} \mathrm{~Pb}+6 \alpha+4 e^{-}+4 \bar{\nu}_{e}+42.8 \mathrm{MeV}
$$

where again a definite number of antineutrinos is emitted and a well defined energy is released. Finally, antineutrinos are also emitted in the $\mathrm{K}$ terminations:

$$
{ }^{40} \mathrm{~K} \rightarrow{ }^{40} \mathrm{Ca}+e^{-}+\bar{\nu}_{e}+1.32 \mathrm{MeV}
$$

The $\bar{\nu}$ energy spectra produced by these three sources are plotted in fig. ??.

Our goal will be to detect antineutrinos emitted by these sources in order to measure the rates of occurrence of the above reactions.

\subsection{Principle of geoneutrino detection}

While low energy neutrino detection offers formidable experimental challenges due to backgrounds as explained above, antineutrinos were discovered in a reaction that naturally affords a nice way to cope with the unwanted background events. The proposed detection reaction:

$$
\bar{\nu}_{e}+p \rightarrow n+e^{+}
$$

is the inverse beta decay (or Reines-Cowan) reaction and generates a positron and a neutron in the final state. The positron gets quickly absorbed in ordinary matter, coupling with an electron and generating two gammas $\left(e^{+} e^{-} \rightarrow \gamma \gamma\right)$ with a total energy release of $1.02 \mathrm{MeV} / \mathrm{c}^{2}$.

The neutron, on the other hand, gets slowed down in the material and finally thermalizes to be absorbed as shown in fig. ??. The lifetime of this process in the Borexino scintillator is of about $200 \mu s$ and at the end the neutron is absorbed by free protons in the scintillator:

$$
H(n, \gamma) D
$$

with the emission of a $2.2 \mathrm{MeV}$ energy gamma.

From the detection viewpoint, this cascade of processes allows a very favorable tag of energies and time.

First of all, the energy of the two electromagnetic cascades are above $\sim 1 \mathrm{MeV}$ and $2.2 \mathrm{MeV}$ respectively. Secondly, the time delay between the two events is a short one, very difficult to mimic by a couple of accidental background events. Finally, these two events are also subjected to a mild spatial condition of coincidence.

In summary, the tagging of an antineutrino event will be an $E>1 \mathrm{MeV} e / \gamma$ event followed (in a narrow time window of, say, $0.5 \mathrm{~ms}$ ) by a $2.2 \gamma$ event. The two events must lie within the typical $(1 \mathrm{~m})$ neutron diffusion length.

The $\bar{\nu}_{e}+p \rightarrow n+e^{+}$detection reaction has a threshold of of $1.8 \mathrm{MeV}$ which is determined by the mass difference between neutron plus positron and the initial state proton. Therefore this reaction has the drawback of not being sensitive to the detection of $\mathrm{K}$ antineutrinos (see fig. ??). 
The detector will reconstruct the antineutrino energy based on the observed positron kinetic energy. The visible energy of the event has to take into account also the $1.02 \mathrm{MeV}$ energy due to annihilation:

$$
E(v i s)=K\left(e^{+}\right)+1.02 M e V
$$

which is plotted in fig. ?? for the case of the $\mathrm{U}$ and Th chain.

In turns, the energy of the antineutrino is shifted with respect to $K\left(e^{+}\right)$by the Q-value of the reaction:

$$
E(\bar{\nu})=K\left(e^{+}\right)-1.8 M e V
$$

So, the final relation between the visible energy and the $\bar{\nu}$ energy is

$$
E(v i s)=E(\bar{\nu})-0.78 \mathrm{MeV}
$$

The observed kinetic energy spectrum E(vis) will begin at $1.02 \mathrm{MeV}$ (the case when the positron kinetic energy is zero).

Apart from the internal (radioactive) contamination of the scintillator, the background to the antineutrino signal can come in principle from a variety of sources, including atmospheric shower particle decays $(\pi, \mu, K)$, relic of past supernovas, non-standard $\nu \rightarrow \bar{\nu}$ oscillations in the Sun, muon induced neutron production and $\bar{\nu}_{e}$ from nuclear reactors.

It can be shown (see ref. [18] and [19]) that the most significant of these external backgrounds is by far the term coming from nuclear reactors.

\subsection{Background from nuclear reactors}

Nuclear power reactors produce energy by fission of heavy nuclei. Since a super-heavy nucleus has a $\sim 30 \%$ excess of neutrons over protons, this excess will be transferred to the lighter fission products which therefore will be beta-instable and produce antineutrinos during their deexcitation.

In order to produce background signal for the inverse-beta decay detection reaction the antineutrinos must have more than $1.8 \mathrm{MeV}$ kinetic energy. The fissile nuclides featuring fission fragments of such energy are ${ }^{235} \mathrm{U},{ }^{239} \mathrm{Pu},{ }^{238} \mathrm{U}$ and ${ }^{241} \mathrm{Pu}$.

The number of fissions generated is typically calculated from the reactor thermal power and the specific fission energy release. In addition, some mild dependence over time is introduced by the initial composition and time evolution of nuclear fuel at a specific reactor.

However, in spite of this parameter variability, antineutrino reactor spectra are all very similar, producing a background that extends from our detection threshold and up to about $9 \mathrm{MeV}$.

For the case of Borexino, the relevant reactors are the ones situated in Europe, which are relatively far away $(700-800 \mathrm{~km}$ ) from the Gran Sasso location (fig. ??). Neutrino oscillation effects will then be washed off in phase giving only an overall reduction factor to about $60 \%$ of the original reactors flux.

Fig. ?? shows both the the reactor background spectrum and the U,Th signal to be searched for. The normalization of the spectra is made accordingly to the expected number of events from european reactors (20 events/year) and the estimated geoneutrino signal in Borexino (6 events/yr). More on this later on.

\subsection{Background from ${ }^{210} \mathrm{~Pb}$}

One of the most important internal backgrounds for the study of geoneutrinos comes from the ${ }^{210} \mathrm{~Pb}$ content of the scintillating material. This nuclide has a $22.3 \mathrm{yr}$ half-life and can be 
introduced in the detecting volume either by natural bulk $\left({ }^{226} \mathrm{Ra}\right)$ contamination or (perhaps most importantly) through ${ }^{222} \mathrm{Rn}$ diffusion.

${ }^{210} \mathrm{~Pb}$ has an $\alpha$-emitting daughter $\left({ }^{210} \mathrm{Po}\right)$ which has a 138 days half-life and can give rise to the reaction

$$
{ }^{13} C(\alpha, n){ }^{16} \mathrm{O}
$$

This originates a chain of events closely mimicking the $\bar{\nu}$ event signal with a first first n-p scattering release (or the $\alpha$ release) followed by neutron capture. In fact this reaction was one of the backgrounds considered in the first detection of geoneutrinos by the Kamland experiment [20].

In the case of the Borexino experiment, the studies conducted in the Counting Test Facility allowed a careful measurement of this background component. In particular ${ }^{210} \mathrm{~Pb}$ was studied during long runs by detecting the ${ }^{210} \mathrm{Po}$ alpha and by studying the total single rate spectra (see fig. ?? for an example of such fits.)

These studies showed that the specific ${ }^{210} \mathrm{~Pb}$ background in Borexino is limited to $\sim 20$ $\mu \mathrm{Bq} /$ ton, a value much lower than the one quoted in [20]. Therefore, this background will be negligible in Borexino.

\section{Antineutrino signal and detection sensitivity}

Having demonstrated that the dominant background in Borexino will be the one due to european reactors, we now proceed to the evaluation of the terrestrial antineutrino signal.

In order to do this, it is necessary to take into account the detailed distribution of U,Th in the Earth's crust, particularly within $500 \mathrm{~km}$ distance from the detector (a region that generates about $1 / 2$ of the total signal).

For this evaluation we have exploited the Earth's model given in [21] which gives U,Th distributions and detailed evluations for specific detector locations. This evaluation, together with the high efficiency (assumed 1) of the detection, leads to the prediction of $\sim 6$ events/yr (oscillation effects included) detected in Borexino, as shown in fig. ??.

\section{Conclusion}

Terrestrial antineutrino detection requires shielded low background detectors of high mass. Borexino, located at Gran Sasso Laboratory, will tackle this fascinating subject armed with its low background capability and its relatively long distance from nuclear reactors.

We estimate that Borexino will detect $\sim 6$ events/yr coming from geoneutrinos with some background from nuclear reactors (fig. ??). The statistical significance of such a signal is predicted to be better than $30 \%$ in 5 years of data taking.

Acknowledgements. We would like to thank Stephen Dye, John Learned and all the organizers of this conference for providing us with a stimulating scientific atmosphere in a wonderful setting.

[1] P.G. Catalano et al., Men. Soc. Geol. It. 35 (1986) 647.

[2] H. Wulandari et al., Astroparticle Physics 22 313-322 (2004).

[3] G. Bellini, Borexino Proposal, 1991, p.184.

[4] G. Alimonti et al., Astroparticle Physics 16 (2002) 205.

[5] C. Arpesella et al., Astroparticle Physics 18 (2002) 1.

[6] G. Bellini (for the Borexino Collaboration), Nucl. Phys. B (Proc. Suppl.) 48 (1996) 363.

[7] G. Alimonti et al., Nucl. Instrum. Meth. A 406 (1998) 411.

[8] G. Alimonti et al., Astroparticle Phys. 8 (1998) 141.

[9] M.Balata et al., Nucl. Instrum. Meth. A 370 (1996) 605.

[10] G.Alimonti et al., Nucl. Instrum. Meth. A 440 (2000)

[11] H.O. Back et al., Physics Letters B 525 (2002) 29 
[12] H.O. Back et al.,Physics Letters B 563 (2003) 37.

[13] H.O. Back et al., JETP Letters 78 (2003) 261.

[14] H.O. Back et al., Physics Letters B 563 (2003) 23.

[15] H.O. Back et al., European Physical Journal C37 (2004) 421.

[16] M. Balata et al., hep-ex/0602027

[17] M. Balata et al., hep-ex/0601035

[18] R.S. Raghavan et al., Phys. Rev. Lett. 80 (1998) 635.

[19] F.Calaprice et al., Geophys. Res. Lett. 25 (1998) 1083.

[20] T. Araki et al., Nature 436 (2005) 499.

[21] F. Mantovani et al., Phys. Rev. D 69 (2004) 013001. 